\title{
Circuit mechanisms underlying memory encoding and retrieval in the long axis of the hippocampal formation
}

Article in Nature Neuroscience · May 2001

DOI: 10.1038/86115 · Source: PubMed

CITATIONS

158

6 authors, including:

Scott Small

Columbia University

220 PUBLICATIONS 15,985 CITATIONS

SEE PROFILE

Robert Delapaz

Columbia University

119 PUBLICATIONS 5,767 CITATIONS

SEE PROFILE

Some of the authors of this publication are also working on these related projects:

Predictive Modeling in Psychiatry and Neurology View project

Project Neuroimaging in malaria View project
READS

151

Gerard Perera

Elekta Inc.

25 PUBLICATIONS 1,778 CITATIONS

SEE PROFILE

Richard Mayeux

Columbia University

1,027 PUBLICATIONS 86,395 CITATIONS

SEE PROFILE 


\title{
Circuit mechanisms underlying memory encoding and retrieval in the long axis of the hippocampal formation
}

\author{
Scott A. Small ${ }^{1,2}$, Arun S. Nava ${ }^{2}$, Gerard M. Perera ${ }^{3}$, Robert DeLaPaz ${ }^{3}$, Richard Mayeux ${ }^{1,2}$ and \\ Yaakov Stern ${ }^{1,2}$
}

The Taub Institute on Alzheimer's Disease and the Aging Brain ${ }^{1}$, and the D epartments of N eurology ${ }^{2}$ and Radiology ${ }^{3}$, Columbia University College of Physicians and Surgeons, 630 West 168 Street, N ew York, New York 10032, USA

Correspondence should beaddressed to S.A.S. (sas68@columbia.edu)

\begin{abstract}
Circuits within the hippocampal formation are active during memory processing. Here we used functional magnetic resonance imaging ( $\mathrm{fM}$ RI) to examine multiple sites across the long axis of the hippocampal formation while subjects performed different phases of an associative memory task, learning to associate faces with names. Viewing faces and hearing names in isolation resulted in separate hippocampal activation patterns. Pairing faces with names resulted a spatially redistributed activation pattern, rather than a simple summation of the activation patterns resulting from viewing faces and hearing names in isolation. Recalling names when cued with faces reactivated a pattern similar to that found during paired training. Finally, the activation patterns representing faces and names were found to be experience dependent, emerging with repeated exposure. Interpreted in the context of hippocampal anatomy and physiology, these findings reveal hippocampal circuit mechanisms that underlie memory encoding and retrieval.
\end{abstract}

The hippocampal formation, a cylindrical structure that spans the longitudinal extent of the medial temporal lobe, is involved in acquiring and retrieving new memories ${ }^{1}$. In contrast to the transverse axis of the hippocampus, the architecture of its longitudinal axis is poorly demarcated, and investigating patterns of connectivity along this axis is technically challenging. Despite the lack of clearly defined anatomical divisions, studies in monkeys ${ }^{2}$ and rats $^{3}$ have shown that the long axis of the hippocampus can be subdivided into functionally distinct regions. Furthermore, there is anatomical ${ }^{4}$ and electrophysiological ${ }^{5}$ evidence that sites across the long axis are interconnected via CA 3 neurons. Thus, the long axis of the hippocampus constitutes a circuit.

Major advances have been made in investigating cellular and molecular mechanisms of plasticity in hippocampal neurons. However, as a structure involved in learning and memory, the hippocampus functions as a network, and establishing how circuits within the network represent and process information is important for understanding how the hippocampus gives rise to memory. Exploring hippocampal circuits is best accomplished by simultaneously examining multiple sites along the circuit's full anatomical extent, and doing so while the circuit is actively en gaged by a cognitive task.

With this goal in mind, we used fM RI to investigate activation patterns across the full longitudinal extent of the hippocampus while subjects performed different phases of a paired-associate memory protocol, associating faces with names. Aside from being clearly hippocampal dependent $t^{6,7}$, paired-associate learning is a memory protocol that lends itself to circuit analysis because the representation of individual items can be discretely established. The manner in which these representations interact during and after paired-associate train- ing can then be determined, thereby exposing circuit mechanisms underlying aspects of associative memory.

We first established that viewing faces and hearing names in isolation are represented by different patterns across thelong axis of the hippocampus, and we characterized the topographic distribution of these patterns. Because the ability to learn an association between separate stimuli requires an intact hippocampus in all species studied-rodents, non-human primates, and humans $5^{6,7}$ - wepredicted that individual hippocampal patterns would somehow interact when subjects were exposed to the pairing of faces with names. Beyond demonstrating that an interaction does in fact occur, our goal was to characterizethesource of the interaction, to offer insight into how the hippocampal circuit functions during memory acquisition.

Studies in both humans and rodents have shown that the hippocampus is not only required for learning associations, but that an intact hippocampal formation is required for recalling information during a limited time window after memory acquisition ${ }^{8,9}$. We thereforealso examined the hippocampal circuit during cued recall. After undergoing paired-associate training (learning to associate a face with a name), subjects viewed faces from the training session and recalled the names associated with each face. Analysis was done to determine whether the cued recall would elicit the same pattern found during the pairing of stimuli.

We next tested whether the hippocampal patterns that represent faces and/or names are experience dependent. This issue surfaced after the results of the first experiment showed that stimuli in isolation or in conjunction have unique hippocampal representations, raising the question of how the hippocampus can accommodate a vast array of data. Similar capacity problems have been solved in other brain areas ${ }^{10}$, in which a neural circuit is coarsely tuned to a source of input, and the representation of 
a
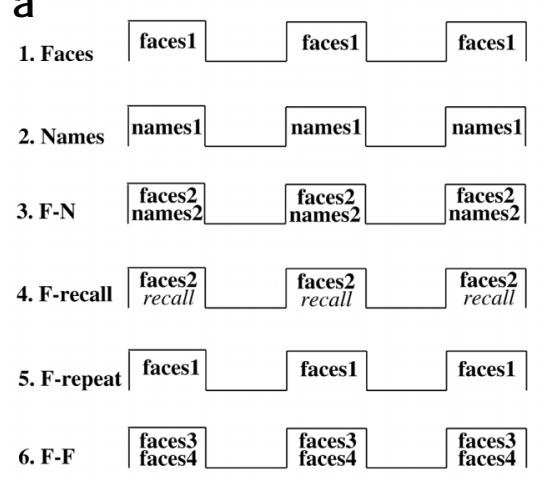

b

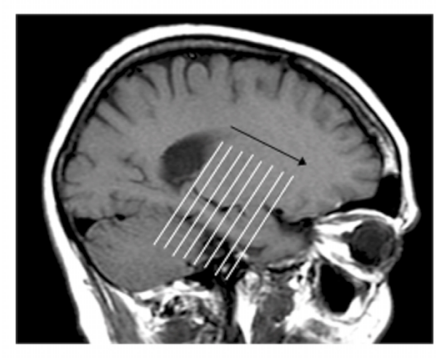

C

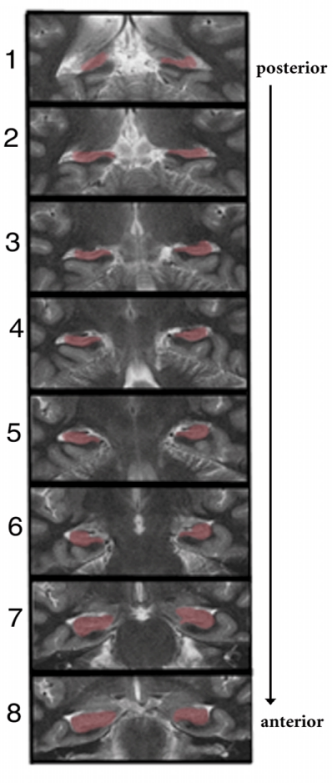

Fig. 1. Experimental design for the three studies. (a) Ten subjects in the first study performed the first four conditions- viewing faces (1), hearing names (2), simultaneously viewing and hearing a new set of faces and a new set of names (3), viewing the second set of faces and recalling the second set of names (4). Ten subjects in the second study performed all conditions including a fifth control condition, viewing the face from the first condition a second time (5). Four subjects in the third experiment performed the first condition and last condition, simultaneously viewing two different faces (6). (b) A sagittal image was used to select multiple transverse slices through the long axis of the hippocampus. (c) Hippocampal RO Is were identified for each of the eight transverse slices, as shown in red. Slices are organized from top to bottom, from posterior hippocampal slice 1 to anterior hippocampal slice 8.

contributes to the binding of the individual items within the pair ${ }^{11}$. We therefore asked whether the hippocampal circuit represents the pairing of stimuli with a pattern that is simply a summation of the patterns for the stimuli in isolation, or whether there is an interaction.

To address this question, we performed a twofactor (condition and hippocampal slice) repeat-

the input is fine tuned with repeated exposure. The purpose of the second experiment was to test for experience-dependent tuning of stimulus representation in the hippocampal circuit.

The series of studies revealed that pairing faces with names resulted in an activation pattern that was not simply the summation of the faces and names patterns in isolation. In addition, pairing faces with names and recalling names when cued with faces resulted in similar activation patterns. The activation patterns representing faces and/or names were found to be experience dependent, emerging with repeated exposure.

\section{RESULTS}

Unique activation topographies for unimodal stimuli

In the first study, 10 subjects performed 4 tasks: viewing a series of 7 unfamiliar faces, hearing a series of 7 low-frequency names, simultaneously viewing and hearing a second series of faces and a second series of names, and viewing the second series of faces while recalling the second series of names (Fig. 1a). Multiple transverse slices were acquired across the longitudinal axis of the hippocampal formation, and regions of interest encompassing the hippocampus in each slice were identified (Fig. 1C). Degree of activation was quantified separately for each condition on an individual basis.

To determine whether the faces-only and names-only conditions were represented by different hippocampal patterns of activation, we performed a two-factor (condition and hippocampal slice) repeated measures M AN OVA, testing for condition $\times$ hippocampal-slice interactions. Results revealed a significant linear interaction between the faces-only and names-only activation patterns $(F=12.3 ; p \leq 0.01)$. Viewing faces resulted in greatest activation in the posterior hippocampus with a posterior-toanterior activation gradient; conversely, hearing names resulted in greatest activation in the anterior hippocampus with an anterior-to-posterior activation gradient (Figs. 2a and 4).

\section{Unique activation topography for paired stimuli}

Paired-associate learning is a fundamental feature of hippocampal function, and it is assumed that the hippocampus actively ed measures MANOVA, which included the faces-names condition (Fig. 1a) and a summated condition generated by adding the faces-only and names-only conditions. The activation pattern associated with the faces-names condition was significantly different from the sums of the unimodal activation patterns, with the greatest difference found in a middle slice of the hippocampus ( $F=11.4 ; p \leq 0.01$; Figs. $\mathbf{2 b}$ and $\mathbf{4}$ ).

\section{Recall reactivated the paired topography}

The hippocampus is involved in associative retrieval for a limited time window after memory acquisition. This provided the rationale for including the fourth condition (Fig. 1a), where subjects viewed the faces from the third condition and were asked to implicitly recall the names associated with each face. A cuedrecall test done outside the scanner found that after paired-associate training, subjects recalled each name with an accuracy ranging from $71-100 \%$ (mean, $90 \%$ ). We reasoned that if the hippocampus was involved in cued retrieval of the paired stimulus (faces-names), viewing faces after training would result in an activation pattern that was similar to the pattern observed during the paired condition.

A two-factor (condition and hippocampal slice) repeated measures M AN OVA was performed including the faces-recall condition (condition 4, Fig. 1a) and the faces-only condition. Recall performance was included as a covariate. The faces-recall condition resulted in a significantly different activation pattern compared to face-only condition, with the greatest difference in middle slices of the hippocampus ( $F=10.2 ; p=0.05$; Figs. $2 \mathbf{b}$ and 4). A direct comparison revealed no significant difference between the faces-names and face-recall conditions ( $F=1.2$ ).

\section{Activation patterns were experience dependent}

The second experiment tested for experience-dependent tuning of the hippocampal patterns encoding faces and/or names. A new group of 10 subjects participated in this study, with a general design similar to the first study (Fig. 1). However, an important difference was that a single stimulus per activation block was used instead of a series of seven separate stimuli, 
Fig. 2. Group data for hippocampal activation patterns associated with each condition from the first study (left) and the second study (right). D ata are organized on the $x$-axis by hippocampal slice, from posterior slice 1 to anterior slice 8. D egree of activation is shown in the $y$-axis reflecting the average number of supra-threshold pixels per slice. (a) Viewing faces in isolation produced a posterior-to-anterior activation pattern, whereas hearing names in isolation produced an anterior-to-posterior activation pattern. (b) Pairing faces with names produced a non-additive activation pattern with greatest activation in the body of the hippocampus. A similar activation pattern was observed when names were recalled cued with faces. (c) Repeat viewing of a face not paired with a name produced a posterior-to-anterior activation pattern that was not different from viewing the face the first time.
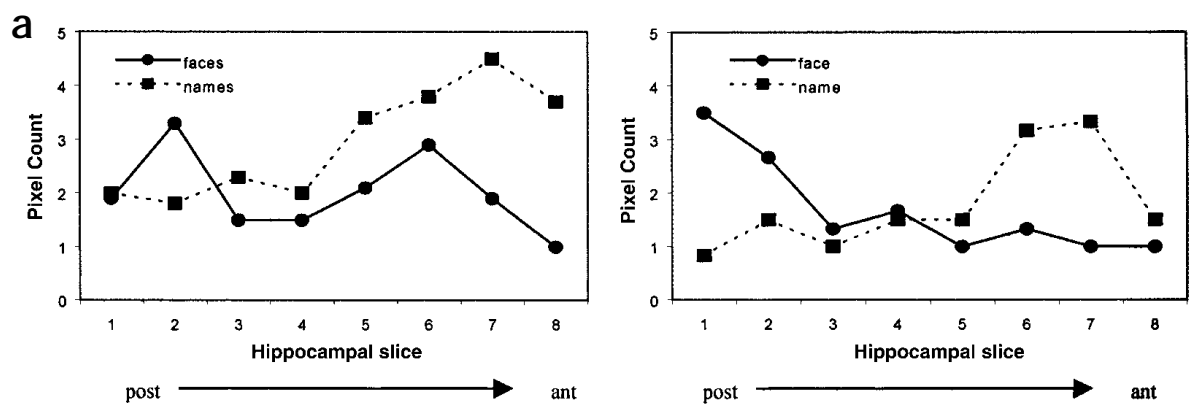

b
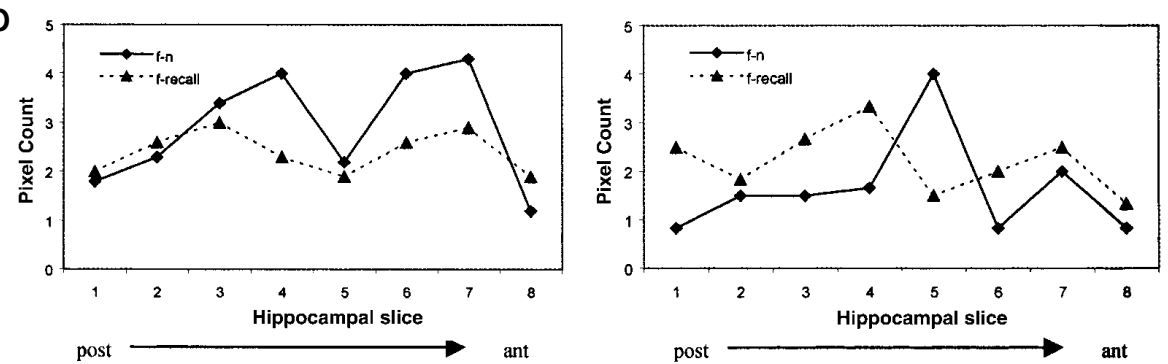

thereby reducing inter-individual variability that occurs for encoding and learning multiple stimuli from block to block. In contrast to the first study, activation patterns were determined separately for individual blocks, by comparing average signal intensity from each block to the average signal intensity of both control blocks.

To test for experience-dependent tuning, a three-factor (condition, slice, block) repeated measures M ANOVA was performed, which included the face-name, face-only and name-only conditions, the eight hippocampal slices, and the first and last blocks. A significant condition $\times$ slice $\times$ block interaction was found ( $F=1.83 ; p \leq 0.05$ ). Post-hoc analyses were done for data from each block. Activation patterns were not significantly different from each other for data acquired during the first block (Fig. 5). During the second block, the face-name activation pattern was not significantly different from the sum of the face-only and name-only conditions, but the face-only and name-only conditions were significantly different from each other $(F=9.96 ; p \leq 0.05)$. During the third block, the face-name activation pattern was significantly different from the sum of the face-only and name-only conditions ( $F=6.91 ; p \leq 0.05)$, and the face-only and name-only conditions were significantly different from each other ( $F=17.48 ; p \leq 0.01$; Fig. 6).

The second study extended and replicated the findings Using data acquired from the third block of the second experiment, we repeated the analyses done in the first study using the same series of M ANOVAs. Data acquired from the third block replicated the findings from the first study (Fig. 2). First, hippocampal activation patterns representing a face and a name in isolation were significantly different from each other ( $F=17.48$; $p \leq 0.01$ ), with the same linear gradient observed in the first study. Second, pairing of a face with a name was represented by a nonadditive pattern, with the greatest activation in the middle of the hippocampus ( $F=6.91 ; p \leq 0.05)$. Third, the recall of a name cued with a face was represented by a pattern with greatest activation in a middle hippocampal slice $(F=10.6 ; p \leq 0.05)$. A direct comparison revealed no significant difference between the facename and face-recall conditions.

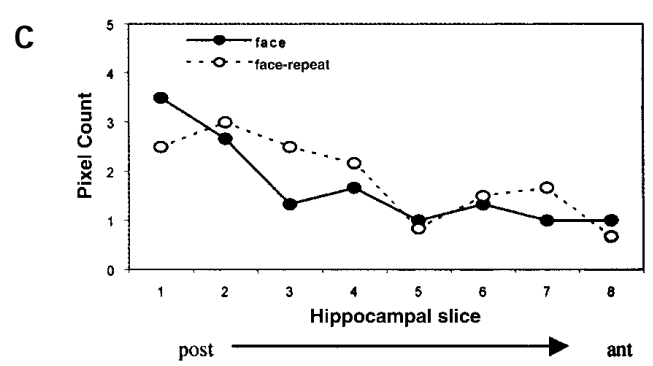

A fifth condition was added to the second study, viewing the same face shown in the first face-only condition a second time (condition 5, Fig. 1a). This condition was designed to test whether the face-recall activation pattern would be elicited by any face after training, and it provided a control for stimulus familiarity. A two-factor (condition and slice) repeated measures M AN OVA was done including the face-only and the facerepeat conditions (conditions 1 and 5, Fig. 1a). The hippocampal pattern found in the fifth condition was not different from the pattern in the first condition ( $F=0.23$; Fig. $\mathbf{2 c}$ ). Thus, the face-recall pattern was not simply related to stimulus familiarity, and seems to be elicited by the specific faces that were used during paired training.

\section{Controlling for stimulus load}

The unimodal and paired conditions were not matched for stimulus load, and the possibility remained that activation in middle hippocampal regions occurred when subjects were exposed to double the amount of information. Results from the second experiment partially addressed this issue: a direct comparison of the face-recall condition and the face-only condition revealed significantly different activation patterns ( $F=5.7 ; p \leq 0.05$; Fig. 7a), and a direct comparison of the first and the third block of the face name condition revealed significantly different activation patterns $(F=6.5 ; p \leq 0.05$; Fig. 7b).

The paired conditions were different from the unimodal conditions both in number of stimuli and in number of modalities. Keeping the number of modalities constant would more precisely test for an effect of stimulus load. We therefore per- 
Fig. 3. Group data from the first and second studies represented by percent change in signal intensity. Data organized as in Fig. 2.

formed a third experiment with four new subjects, in which stimulus load was varied while modality was held constant. In the first task, subjects viewed a series of faces shown individually (condition 1, Fig. 1a), and in the second task, subjects viewed pairs of new faces (condition 6 , Fig. 1a). In both cases, greatest activation was observed in posterior hippocampal slices (Fig. 7c). A MANOVA comparing these two conditions revealed no difference between these conditions.
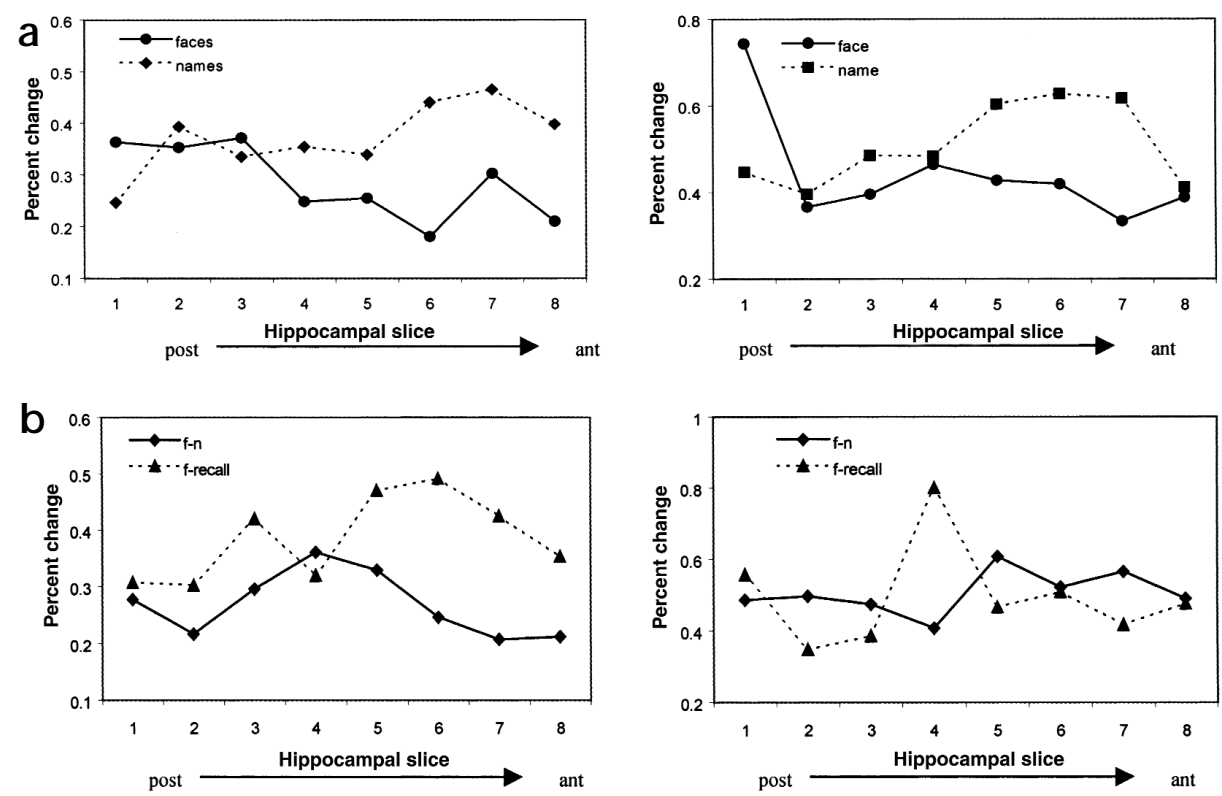

\section{Discussion}

Since implicated as a brain region

involved in memory function almost 50 years ago, parallel lines of investigation have focused on mapping the functional anatomy of the hippocampus and surrounding structures, and on determining cellular mechanisms of plasticity in hippocampal neurons. A more recent focus has been on investigating circuit mechanisms within the hippocampal network in an effort to account for its mnemonic abilities.

Here we use fM RI to investigate the hippocampus as a circuit. Because of the vascular organization of the hippocampus ${ }^{12}$, the longitudinal axis of the hippocampus is particularly amenable to BOLD fM RI analysis. An advantage of fM RI over electrophysio-
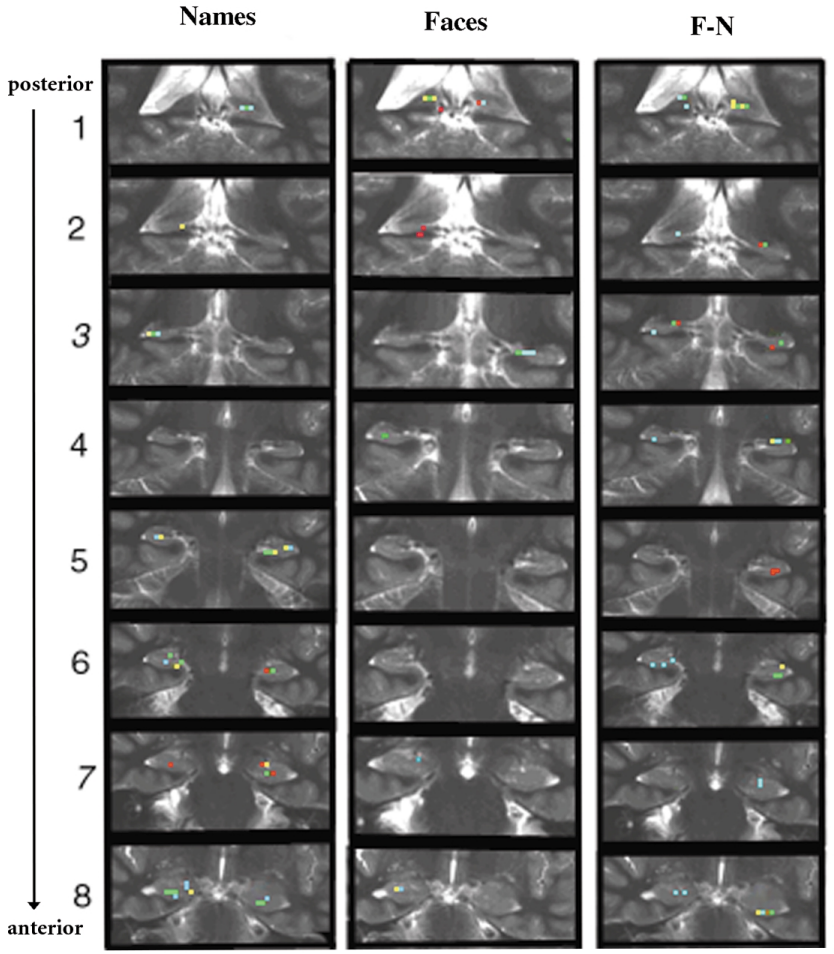

F-recall

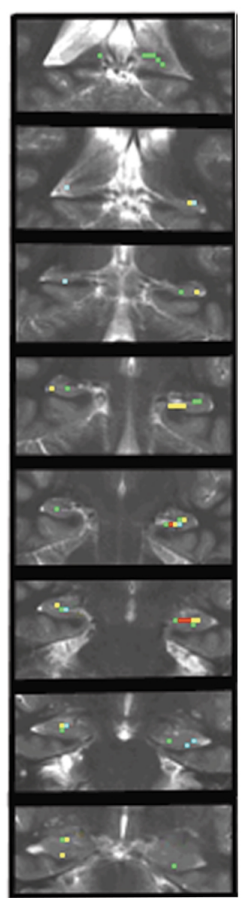

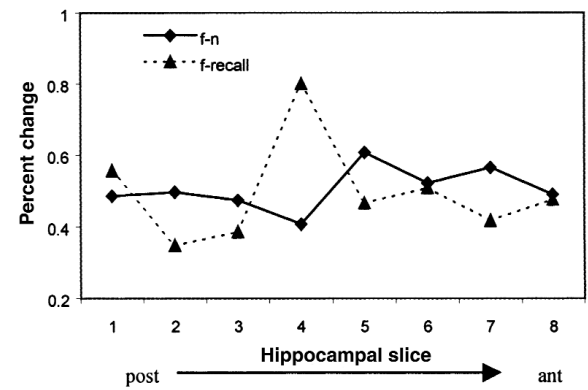

C

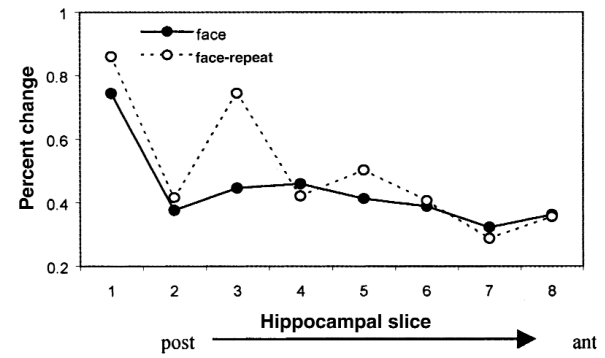

logical techniques is that it can simultaneously examine multiple sites across large brain volumes, as illustrated in the current study, in which the full longitudinal extent of the hippocampus was assessed while subjects performed memory tasks. Using this approach, a number of mechanisms have been revealed that can help explain how the hippocampal circuit encodes simplestimuli, and how it processes complex stimuli during learning and recall.

First, we found that the hippocampal formation encodes different stimuli- viewing faces and hearing names- with unique topographical representation across its long axis. Second, we found that these representations emerge with repeated exposure. Taken together, these findings provide evi-

Fig. 4. Hippocampal activation patterns associated with each condition; an individual example from the first study. Slices are organized from top to bottom, from posterior hippocampal slice 1 to anterior hippocampal slice 8 . Activated pixels within each hippocampal RO I are color coded (blue $z \geq 1.96$; green $z \geq 3.4$; yellow $z \geq 4.8$; red $z \geq 6.2$ ). 
a

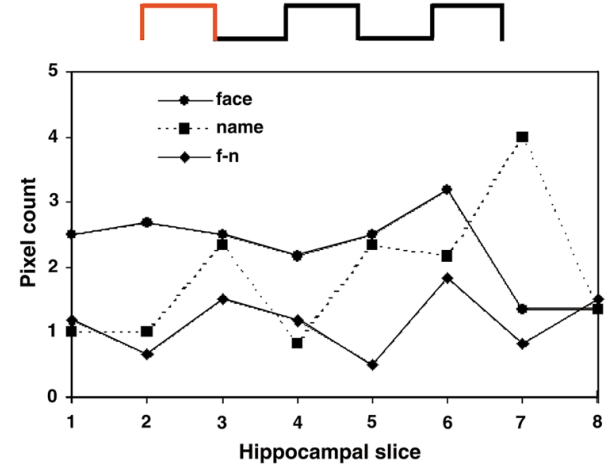

b

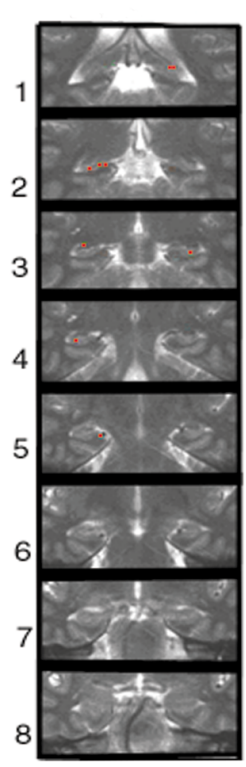

Name 1st

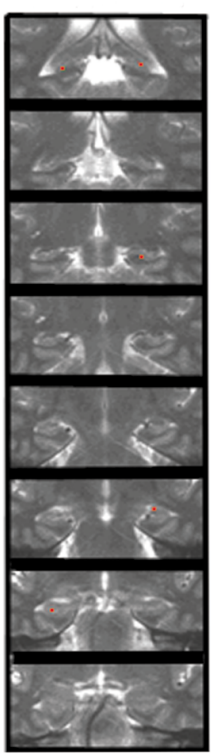

F-N 1st

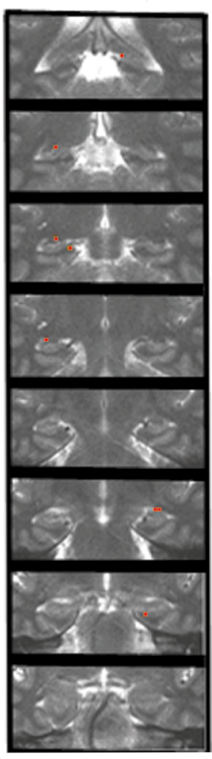

dence for how the hippocampus can index input from various neocortical sites, and how it can do so for a vast array of data. Because the ultimate storage site of long-term memory resides in the neocortex, the hippocampus must be able to re-access the same neocortical sites that provided the initial input. The different activation patterns observed for faces and for names assign unique topographical addresses within the hippocampus, which can connect with different neocortical regions encoding faces or names.

The hippocampal formation receives input from diverse neocortical regions, consistent with the behavioral observation that the hippocampus is involved in learning to remember information across different modalities. The neocortex and the hippocampus are indirectly connected via the parahippocampal gyrus. Neocortical input onto the parahippocampal gyrus is topographically arranged, such that anterior neocortical regions connect to anterior parahippocampal sites, and posterior neocortical regions connect to posterior parahippocampal sites ${ }^{13,14}$. Limit-

Fig. 6. Results from the second study showing that activation patterns measured during the third block were more finely tuned to faces and/or names. (a) Group data showing that viewing a face and hearing a name produced separate activation patterns, and that pairing faces with names produced a non-additive activation pattern. (b) An example from a single subject.

Fig. 5. Results from the second study showing that activation patterns measured during the first block were coarsely tuned to faces and/or names. (a) Group data showing no significant difference among the patterns. (b) An example from a single subject.

ed anatomical studies suggest that this topography may be preserved in the input the hippocampus receives from the parahippocampal gyrus ${ }^{15}$. The topographical organization is not as restrictive as the topographical mapping in primary neocortex; rather, it consists of overlapping fields forming a 'topographical gradient'16. This type of low-fidelity topography provides an anatomical substrate for the functional maps observed in the current study, where early activation patterns were coarsely tuned to different stimuli.

Dynamic physiological properties of subregions within the hippocampus can account for the experience-dependent tuning observed. Stimulating neurons in the entorhinal cortex, the main parahippocampal gateway into the hippocampus, first activates subregions al ong the transverse axis of the hippocampus 5 . Because CA 3 neurons are interconnected in the longitudinal axis, entorhinal stimulation will al so secondarily activate subregions in longitudinally distal sites. Timing requirements for the induction of long-term plasticity ${ }^{17}$ pre

a

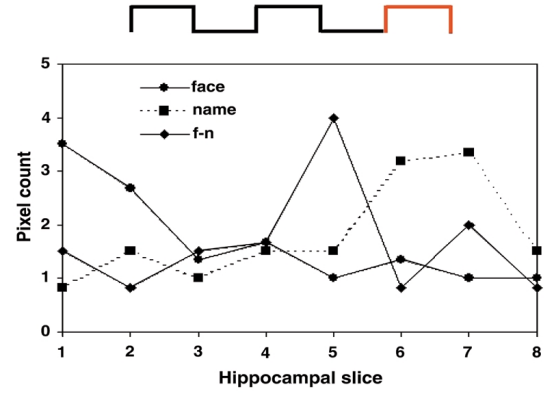

b
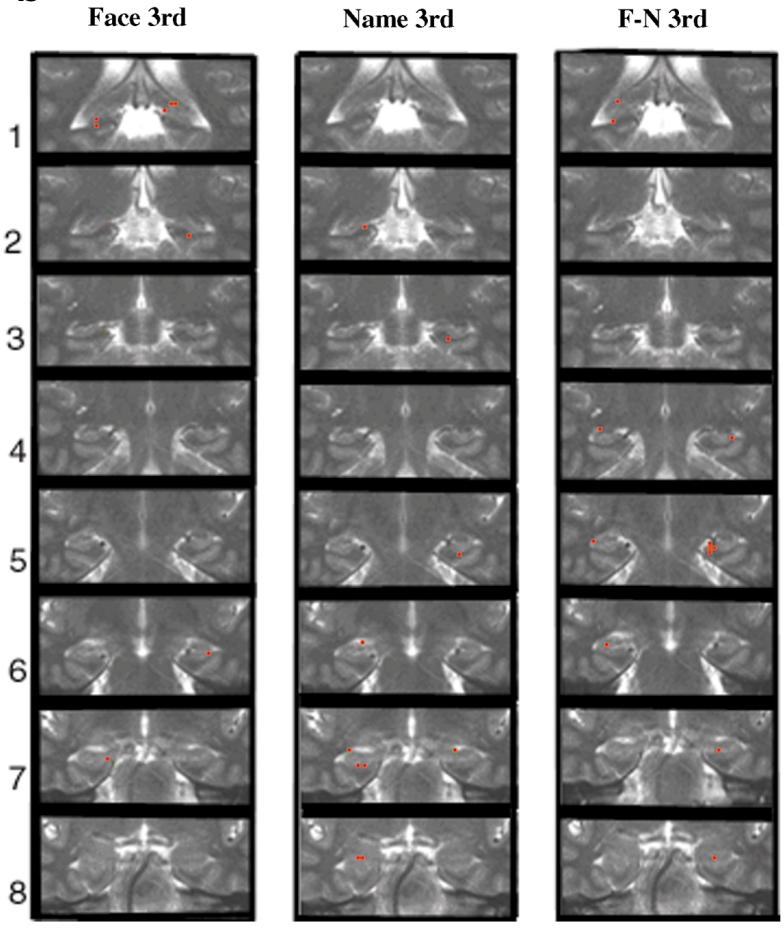

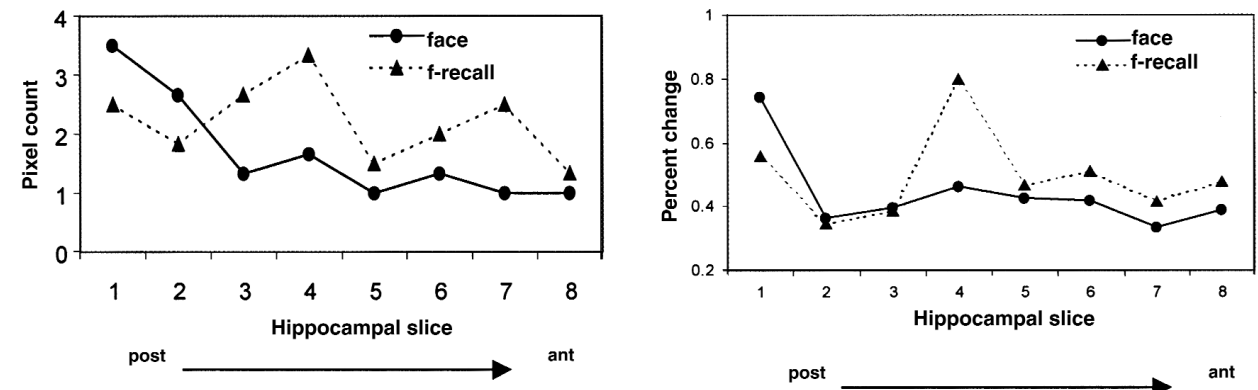

b
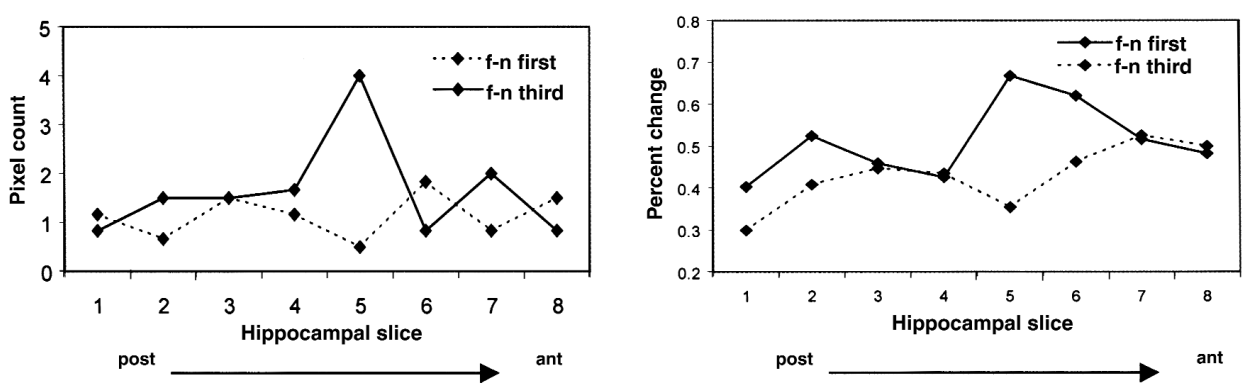

C
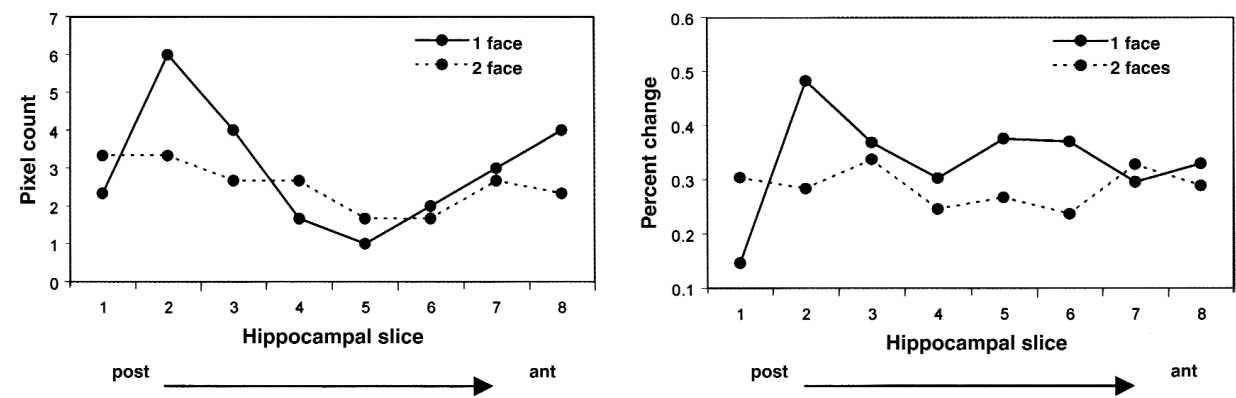

Fig. 7. Results from the second and third experiments that control for stimulus load shown with pixel counts (left) and with percent change in signal (right). (a) The face-recall and face-only conditions were matched for stimulus load, but produced different activation patterns. (b) The paired conditions in the first and third blocks were matched for stimulus load, but produced different activation patterns. (c) The two conditions in the third experiment- viewing one or two faces- were matched for stimulus modality and unmatched for stimulus load, but produced similar activation patterns.

dict that repeated volleys of entorhinal stimulation would differentially strengthen connections between entorhinal neurons and hippocampal subregions within the same transverse slice. Thus, the anatomy of the hippocampus together with cellular mechanisms of hippocampal neurons can mediate the experience-dependent focusing observed in the current study.

The third finding of this study showed that the pairing of stimuli resulted in a hippocampal activation pattern that could not be accounted for by a simple summation of the patterns found for the stimuli in isolation. The fact that non-additivity was reflected by spatial redistribution of the activation pattern, and not only by changes in number of supra-threshold pixels per slice, suggests the occurrence of spatial integration. A specific circuit mechanism underlying spatial integration is suggested by the patterns observed within the hippocampus during the unimodal and paired conditions. The activation patterns of the faces-only and names-only conditions were best modeled as linear functions with reverse slopes (Figs. 2a and $6 \mathbf{6}$ ), and these patterns spatially convergein middle hippocampal slices. Thus, the results suggest that the hippocampus may function as a convergence zone for data that are encoded in anatomically distant sites in the neocortex, and that the hippocampus actively contributes to forming associations. In contrast to the experience-dependent tuning that might be governed by plasticity in the transverse circuit, the activation pattern that emerges with the pairing of separate incoming stimuli is more likely to be governed by CA3-CA3 plasticity ${ }^{18}$. The third experiment was designed to address concerns about stimulus load. Nevertheless, an event-related design would provide a more direct control by comparing activation patterns of paired items that were successfully and unsuccessfully learned.

Although studies have shown that the hippocampal formation is involved in memory retrieval, few studies have assessed how the hippocampus functions as a circuit during retrieval. In the absence of empirical evidence, computational models have suggested a mechanism of pattern completion: when cued with a memory fragment, the circuit pattern of the full memory is reactivated ${ }^{19,20}$. The final finding of this study supports this mechanism. After training, when subjects viewed a face - a fragment of the paired stimulus - reactivation of a similar, though not identical, paired pattern was observed. 
Another functional imaging study has demonstrated hippocampal activation during associative learning ${ }^{21}$, and a number of studies have explored activation along the anterior-posterior axis of the hippocampus during different aspects of memory processing 22,23. A number of procedural differences exist between our study and other fM RI studies investigating the hippocampal formation during memory processing. For example, we focused our analysis exclusively on the hippocampus proper, and excluded the parahippocampal gyrus. Nevertheless, our findings for visual information generally align with other studies. Specifically, encoding visual information resulted in greatest activation in the posterior hippocampus ${ }^{24}$, whereas retrieval resulted in activation in relatively more anterior hippocampal regions ${ }^{25}$. Although the use of auditory stimulation is less common, one fM RI study found that hearing novel words resulted in greatest activation in the anterior hippocampus ${ }^{26}$.

There are, however, a number of apparent inconsistencies between our observations and those made in other functional imaging studies. For example, although the current studies were not designed to test this, no laterality effect during the encoding of faces was detected in preliminary analysis. This is likely due to the procedures used in the current study. Specifically, subjects were not explicitly instructed to memorize the faces, and passive encoding of faces results in a diminished laterality of medial temporal lobe activation compared to intentional memorizing of faces ${ }^{27}$. Furthermore, the relatively long exposure time of each face increases the likelihood that subjects used both visual and verbal encoding strategies. Demonstrating that repeated exposure of the same stimulus results in changes in the hippocampal activation pattern - as observed in the second experiment of the current study-does not necessarily contradict other studies that use repeated stimuli as a control condition 24,25,28. In our study, stimuli were shown 4 times per block for a total of 12 exposures, significantly fewer exposures than in other studies.

\section{MethODS}

Subjects. A total of 24 subjects participated in the three studies: 10 subjects each in the first and second experiments, and 4 subjects in the third experiment. All subjects were healthy and were between 22 and 35 years old. The institutional review board at Columbia University School of Physicians and Surgeons approved the experiments, and subjects provided informed consent to participate.

Data acquisition. In the first study, subjects were scanned while performing the first four activation conditions (Fig. 1a). All conditions had the same blocked design, with three $36-s$ activation phases alternating with two 36-s baseline phases consisting of viewing cross hairs, for a total task duration of three minutes. During each activation phase, seven separate items (faces, names or faces-names) were presented sequentially, with a presentation duration of $4.5 \mathrm{~s}$ per item and an ITI of $0.5 \mathrm{~s}$.

In the second study, subjects were scanned while performing the five activation conditions (Fig. 1). All conditions had the sameblock design, with a 20-s activation phase alternating with a 20-s baseline phase. During each activation, a single stimulus (face, name or face name) was presented sequentially four times, with a presentation duration of $4.5 \mathrm{sper}$ item and an ITI of 0.5 seconds. The design of the first and last conditions (Fig. 1a) used for the third study was identical to the design used in the first study.

Scanning was done using a GE 1.5-tesla magnetic resonance scanner retrofitted for echo planner imaging. A sagittal scout image was used to select 5-mm oblique-coronal slices perpendicular to the long axis of the hippocampal formation. A gradient echo sequence (TE/TR/flip angle, $45 / 3000 / 90$ ) and a standard quadrature head coil was used to acquire T2*-weighted images with an inplane resolution of $2.3 \times 2.3 \mathrm{~mm}$. Fast Multiplanar Inversion Recovery images (TE/TR/TI $=43 / 6500 / 200$ ) with an inplane resolution of $0.29 \times 0.29 \mathrm{~mm}$ were also acquired to provide high resolution structural images of the regions of interest. The AIR pro- gram ${ }^{29}$ was applied to correct for head motion and co-register the volumes. I mages were not spatially filtered.

D ata analysis. For single-subject analysis, eight slices were selected that covered the full long axis of the hippocampal formation (Fig. 1b). Fixed anatomical criteria ${ }^{30,31}$ were used to identify hippocampal regions of interest (ROIs), excluding the entorhinal cortex, for each of the eight slices. Pixel-by-pixel t-tests were done and percent increase in signal was determined by comparing signal intensity acquired during activation and baseline phases, excluding the first $10 \mathrm{~s}$ of each phase. Resultant statistical maps were co-registered onto the high-resolution images.

For the first study, averagesignal intensity was computed from all three activation blocks; for the second study, average signal intensity was computed separately for each activation block. A count of above-threshold pixels per hippocampal slice was used to quantify activation in each ROI and was used in group data analysis. Pixel count was preferred over percent increase in signal because of the anatomical inhomogeneity of the hippocampal ROIs; nevertheless, group data are shown using both pixel count and percent change in signal.

For group data analysis, a two within-factor repeated measures M AN OVA was constructed, in which experimental conditions constituted the levels of the first factor, and the eight hippocampal slices constituted the levels of the second factor. Preliminary investigation revealed no clear laterality effect, and counts from the left and right hippocampi were aggregated. Polynomial or deviation contrasts were applied to the eight levels of the slice factor. The interaction of condition $\times$ slice was the most important outcome measure of the model, because this reflected hippocampal activation patterns, and interaction contrasts were constructed from the single factor contrasts.

\section{ACKNOWLEDGements}

Wethank B. Rakitin for his assistance in data analysis. This work was supported in part by federal grants AG 08702 and AG 00946, the Beeson Faculty Scholar Award from the American Federation of Aging and the Charles S. Robertson gift from the Banbury Fund.

\section{Received 14 November 2000; AcCepted 29 January 2001}

1. Squire, L. R. Memory and the hippocampus: a synthesis from findings with rats, monkeys, and humans. Psychol. Rev. 99, 195-231 (1992) [erratum, Psychol. Rev. 99, 582, 1992].

2. Colombo, M., Fernandez, T., Nakamura, K. \& Gross, C. G. Functional differentiation along the anterior-posterior axis of the hippocampus in monkeys. J. Neurophysiol. 80, 1002-1005 (1998).

3. Moser, M. B. \& Moser, E. I. Distributed encoding and retrieval of spatial memory in the hippocampus. J. Neurosci. 18, 7535-7542 (1998).

4. Amaral, D. G. Emerging principles of intrinsic hippocampal organization. Curr. Opin. Neurobiol. 3, 225-229 (1993).

5. Pare, D. \& Llinas, R. Non-lamellar propagation of entorhinal influences in the hippocampal formation: multiple electrode recordings in the isolated guinea pig brain in vitro. Hippocampus 4, 403-409 (1994)

6. Eichenbaum, H., M athews, P. \& Cohen, N. J. Further studies of hippocampal representation during odor discrimination learning. Behav. Neurosci. 103, 1207-1216 (1989).

7. Vargha-Khadem, F. et al. Differential effects of early hippocampal pathology on episodic and semantic memory. Science 277, 376-380 (1997) [erratum, Science 277, 1117, 1997]

8. Kim, J. J. \& Fanselow, M. S. Modality-specific retrograde amnesia of fear. Science 256, 675-677 (1992)

9. Rempel-Clower, N. L., Zola, S. M ., Squire, L. R. \& Amaral, D. G. Three cases of enduring memory impairment after bilateral damage limited to the hippocampal formation. J. Neurosci. 16, 5233-5255 (1996).

10. Debski, E. A., Cline, H. T. \& Constantine-Paton, M. Activity-dependent tuning and the N M DA receptor. J. Neurobiol. 21, 18-32 (1990).

11. Marr, D. Simple memory: a theory for archicortex. Phil. Trans. R. Soc. Lond. B Biol. Sci. 262, 23-81 (1971)

12. Anderson, P., Bliss, T. V. \& Skrede, K. K. Lamellar organization of hippocampal pathways. Exp. Brain Res. 13, 222-238 (1971).

13. Insausti, R., Amaral, D. G. \& Cowan, W. M. The entorhinal cortex of the monkey: Il. cortical afferents. J. Comp. Neurol. 264, 356- 395 (1987).

14. Suzuki, W. A. \& Amaral, D. G. Perirhinal and parahippocampal cortices of the macaque monkey: cortical afferents. J. Comp. Neurol. 350, 497-533 (1994).

15. Witter, M. P. \& Amaral, D. G. Entorhinal cortex of the monkey: V. projections to the dentate gyrus, hippocampus, and subicular complex. J. Comp. Neurol. 307, 437-459 (1991) 
16. Witter, M.P., Groenewegen, H.J., Lopes da Silva, F.H \& Lohman, A.H. Functional organizationof the extrinsic and intrinsic circuitry of the parahippocampal region. Prog. Neurobiol. 33 161-253 (1989)

17. Gustafsson, B., Wigstrom, H., Abraham, W. C. \& Huang, Y. Y. Long-term potentiation in the hippocampus using depolarizing current pulses as the conditioning stimulus to single volley synaptic potentials. J. Neurosci. 7, 774-780 (1987).

18. Pavlidis, P., M ontgomery, J. \& Madison, D. V. Presynaptic protein kinase activity supports long-term potentiation at synapses between individual hippocampal neurons. J. Neurosci. 20, 4497-4505 (2000).

19. Kohonen, T. Associative M emory-A System-Theoretical Approach (Springer, Berlin, 1977).

20. McClelland, J. L. \& Rumelhart, D. E. Distributed memory and the representation of general and specific information. J. Exp. Psychol. Gen. 114 159-197 (1985)

21. Henke, K., Buck, A., Weber, B. \& Wieser, H. G. Human hippocampus establishes associations in memory. Hippocampus 7, 249-256 (1997)

22. Schacter, D. L. \& Wagner, A. D. M edial temporal lobe activations in fM R and PET studies of episodic encoding and retrieval. H ippocampus 9, 7-24 (1999).

23. Lepage, M., Habib, R. \& Tulving, E. Hippocampal PET activations of memory encoding and retrieval: the HIPER model. Hippocampus 8, 313-322 (1998).
24. Stern, C. E. et al. The hippocampal formation participates in novel picture encoding: evidence from functional magnetic resonance imaging. Proc. $\mathrm{N}$ atl. Acad. Sci. USA 93, 8660-8665 (1996).

25. Gabrieli, J. D. E., Brewer, J. B., Desmond, J. E. \& Glover, G. H. Separateneural bases of two fundamental memory processes in the human medial temporal lobe. Science 276, 264-266 (1997).

26. Saykin, A. J. et al. Functional differentiation of medial temporal and frontal regionsinvolved in processing novel and familiar words: an fM RI study. Brain 122, 1963-1971 (1999).

27. Kelley, W. M. et al. Hemispheric specialization in human dorsal frontal cortex and medial temporal lobe for verbal and nonverbal memory encoding. Neuron 20, 927-936 (1998)

28. Kirchhoff, B. A., Wagner, A. D., M aril, A.\& Stern, C. E. Prefrontal-temporal circuitry for episodic encoding and subsequent memory. J. Neurosci. 20, 6173-6180 (2000)

29. Woods, R. P., Grafton, S. T., Watson, J. D., Sicotte, N. L. \& Mazziotta, J. C. Automated image registration: II. Intersubject validation of linear and nonlinear models. J. Comput. Assist. Tomogr. 22, 153-165 (1998).

30. Duvernoy, H. M. TheH uman H ippocampus; An Atlas of Applied Anatomy (J. F. Bergman, Munich, 1998).

31. Amaral, D. G. \& Insausti, R. in The Human Nervous System (ed. Paxinos, R.) 711-755 (Academic, San Diego, 1990). 